\title{
Autonomous Learning in Vertical Environments
}

\author{
Professor Matthew Cusack and Dr. Terence Fogarty
}

${ }^{2}$ Faculty of the Built Environment, University of the West of England, Coldharbour Lane, Frenchay, Bristol BS16 1QY, Great Britain

${ }^{b}$ Bristol Transputer Centre, Faculty of Computer Studies and Mathematics, University of the West of England, Coldharbour Lane, Frenchay, Bristol BS16 1QY, Great Britain

\section{Abstract}

This paper describes the further development of earlier work on wall climbing robots and recent research on the development of control systems, to enable the robots to learn the best strategies for climbing and carrying out various inspection and maintenance tasks on civil engineering and building structures.

There are three main strands to this research: a rule-based learning system, the wall climbing robots, and the integration of the two into an autonomous, adaptive system.

\section{INTRODUCTION}

The aim of this research is to design a rule-based learning system to support the various kinds of adaptation useful to autonomous systems in general and climbing robots in particular, i.e. evolution, learning and programming. Rule-based learning systems have been built that can achieve one or two of these kinds of adaptation on a particular simulated task. To date, no one has built a system to achieve all of these and they have not as yet been used on real systems.

The rule-based learning system to control a climbing robot will be programmed with rules enabling it to:-

i. recognise the various surfaces it will encounter via its sensors,

ii. constrain its actions to those which are both safe and sensible,

iii. have a goal towards which it can strive,

iv. measure how well it is doing.

Thus an interface between rules at the programming level and rules at the learning level will be provided. It will also be capable of discovering new rules and presenting these to a human being. 


\section{RULE-BASED LEARNING SYSTEMS}

Rule-based learning systems [1] lie midway between neural networks and symbolic processing systems and combine the benefits of both. They are massively parallel, message passing, rule-based systems which use genetic algorithms $|2|$ to discover new rules as well as providing for reinforcement learning and programming. It has been proposed that a suitable application of genetic algorithms is to evolve robots [3]. The most suitable way to use genetic algorithms to evolve the control systems for real robots is within the rich framework provided by rule-based learning systems.

Autonomous control systems need to be able to adapt to circumstances, i.e. to weigh up a number of strategies based on how well they are working and to choose the best amongst them with high probability. So appropriate reinforcement algorithms are being used. The standard reinforcement learning algorithm for rule-based learning systems is the bucket brigade [ $\{$ ] but the interaction between this and the genetic algorithm is complicated and has not been shown to work reliably on any system. It is proposed that the genetic algorithm be decoupled from the reinforcement learning algorithm and that a number of other algorithms be looked at to provide the reinforcement component of the rule-based learning system. These will include back-propagation and temporal difference methods.

This process of adaptation must work within the constraints of programmed and learned rules. It is this interaction between evolution, learning and programming that must be resolved to allow the genetic algorithms to be used on real systems.

At a recent workshop on learning systems [5] a number of groups presented successful applications of the genetic algorithm to control problems. However, one cannot evolve complex systems with a simple genetic algorithm nor is it wise or safe to start from scratch in real applications where programmed knowledge can provide constraints for the genetic algorithm to work within it [6]. If the genetic algorithm is to be used to evolve systems for industrial |7| or commercial |8| applications this is best done in the framework provided by rule-based learning systems. These:-

i. can be programmed,

ii. can learn rules from examples,

iii. can weight rules by reinforcement such as provided by reinforcement learning algorithms,

iv. can discover new rules using parallel genetic algorithms which allow both co-evolution and growth to take place.

\section{WALL CLIMBING ROBOTS}

Prototype wall climbing robots that can be used as test beds for a range of inspection and maintenance end effectors are being designed and constructed. Each robot will:-

i. be able to travel quickly to the points requiring inspection, 
ii. be able to move in smaller steps when using measuring equipment,

iii. turn, incrementally, on its own axis,

iv. have as high a power to weight ratio as possible so that reasonable payloads can be carried and to ensure that the robot is as stable as possible when using the tools and end effectors fitted to it,

v. enable the tools and measuring equipment to be easily fitted and interchanged,

vi. be able to check the feet for vacuum.

The robots will be able to move over the surfaces of buildings and be able to perform tests on the structure and carry out remedial work. The design will aim at the following target group of structures:-

i. a typical motorway bridge

ii. a Yorkon type office building

iii. a multi-storey residential building

It will be possible to transfer the basic concept to other robots in this field. The cost of designing a robot for a specific building will be small compared to the capital cost of the building itself. The main thrust of the project will be to develop the enabling technology which can be easily transferred to a variety of structures. Sites at Bristol and London have already been identified for test purposes. Initially, a small-scale prototype will be built to:-

i. test various feet configurations and develop climbing strategies,

ii. test a new concept in vacuum feet which will conform to most of the surfaces in use in construction,

iii. have a high degree of mobility, i.e. be able to move up and down surfaces by rotating at either $5^{\circ}$ or $90^{\circ}$ to traverse building structures including overhanging projections,

iv. act as a test bed to further the work already carried out at the University on testing building structures,

v. develop the technology to carry out the remedial work required on the test structures.

When the small-scale trials are in an advanced stage, a full-scale robot will be built and tested on the selected, previously referred to, test sites. The following test methods will be used:- 
i. visual

ii. rebar

iii. carbonation

iv. chlorination

v. rebar corrosive

vi. dalamation

vii. voids.

Two wall climbing robots have been built at Bristol Polytechnic. The first -Sasquatch |12| created considerable interest at the May 1991 Automan Exhibition at the National Exhibition Centre.

The second - Sysiphus [13] - was designed to carry instrumentation for measuring defects in structures and tools for carrying out remedial work. (Figure 1)
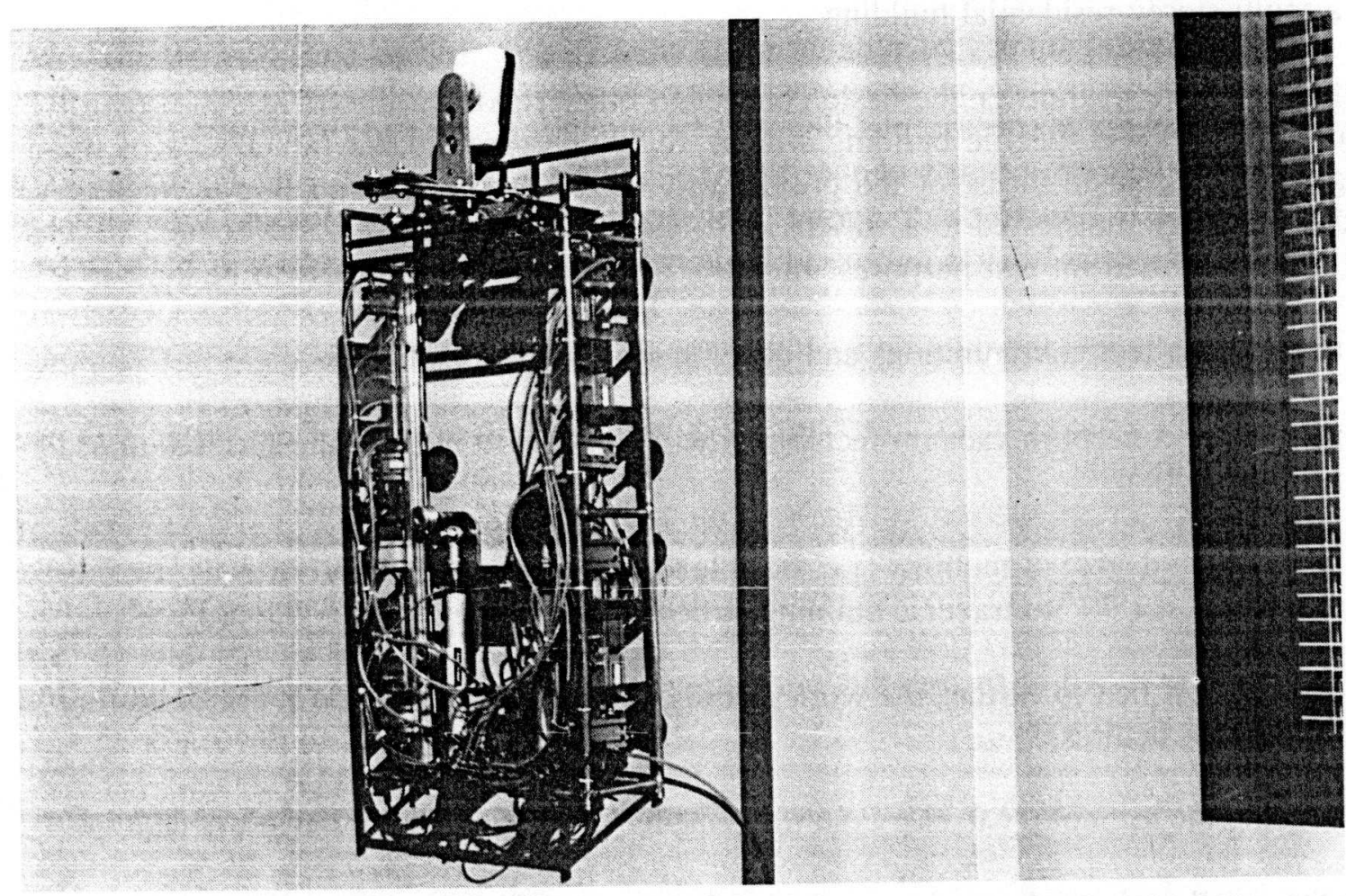

Figure 1. Sysiphus 
This robot is controlled by software written in $\mathrm{C}++$ on a PC and downloaded to the target system using Sourceview ${ }^{\mathrm{TM}}$. The target system is based on a $80 \mathrm{C} 188$ microprocessor running on an STE bus. The design is based on a sliding vacuum foot attached to a deck which can be raised and lowered relative to the main frame using pneumatically operated cams. The target processor then controls the robot movement through its parallel input/output (PIO) interface. The PIO in turn opens and closes the $12 \mathrm{v}$ solenoid valves allowing control of the pneumatic system and ejectors used to create vacuum for the feet.

The main frame contains the cam mechanism and has small feet attached to it, thus raising or lowering the cams, either a big foot or small feet are in contact with the surface to be traversed. The robot is capable of forward, reverse motion in a large step mode, and inching forward motion in small step mode, rotation in either clockwise or anticlockwise direction by 5 degrees per step. The platform may be parked with all twelve feet adhering to the surface, which gives a very stable platform from which to operate monitoring equipment.

\section{INSPECTION AND TESTING}

Some preliminary work has already been undertaken to identify appropriate testing techniques. A lightweight mechanism, operated by a pneumatic piston mounted on a supplementary frame parallel to the surface being measured, has been developed. This has been used in tests undertaken using a standard cover meter (Figure 2). Preliminary trials indicate that this method can produce reliable information on rebar cover and concrete cover depth.

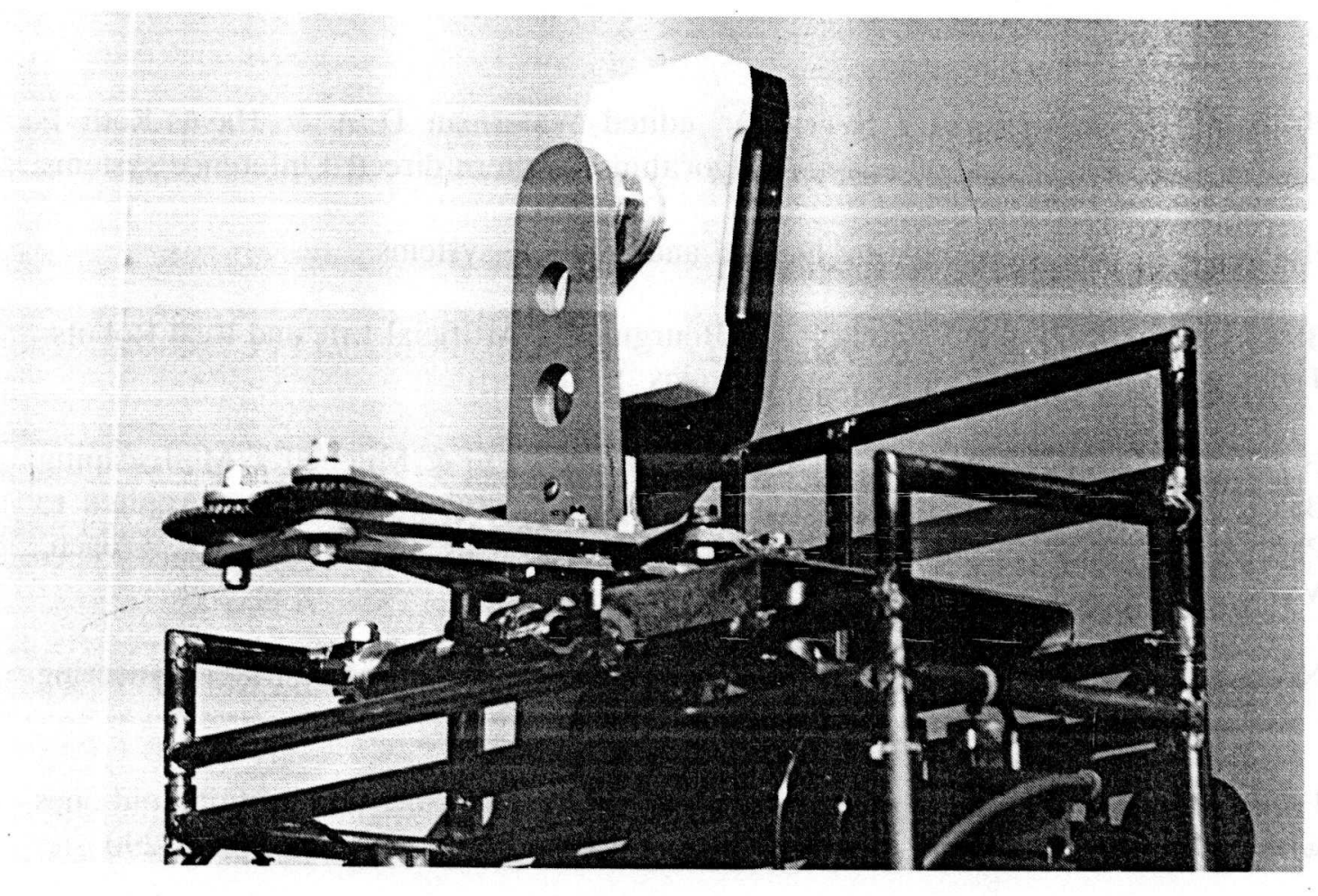

Figure 2.

Cover Meter 
A feasibility study $19 \mid$ undertaken for the DTI by CIRIA - the Construction Industry Research and Information Association - between May 1986 and July 1987 identified the inspection of civil engineering and building structures as having the greatest potential for developments in automation and robotics. Providing access has been identified as the major component in the total cost of inspection [10]. Safety statistics [11] for the period 1981 to 1985 showed that maintenance activity accounted for between $34 \%$ and $50 \%$ of the total number of fatal accidents in construction and that of these $30 \%$ were caused by falls from scaffolds with $20 \%$ as a result of falls from ladders. Apart from the human factor, accidents of this nature have substantial financial consequences for the industry.

\section{CONCLUSIONS}

The successful development of prototype wall climbing robots is now at an advanced stage. Initial research into the potential use of neural networks and more specifically rule-based learning systems control looks extremely promising.

Autonomous control systems need to be able to adapt to circumstances, i.e. to weigh up a number of strategies based on how well they are working and to choose the best amongst them with high probability, so appropriate reinforcement algorithms are being used. It is proposed that the genetic algorithm be decoupled from the reinforcement learning algorithm and a number of other algorithms be looked at to provide the reinforcement component of the rule-based learning system. It is the interaction between evolution, learning and programming that must be resolved to allow the genetic algorithms to be used on real systems.

\section{REFERENCES}

[1] Holland J H \& Reitman J S (1978) edited Waterman D A \& Hayes-Roth F Cognitive systems based on adaptive algorithms. Pattern directed inference systems

[2] Holland J H (1975) Adaptation Natural and artificial systems

131 Brooks R A (1992) (eds) Varela F J \& Bourgine P Artificial Life and Real Robots. Towards a Practice of Autonomous Systems p3-10

[4] Holland J H (1987) eds. Michalski R S, Carbonell J G \& Mitchell T M Escaping Brittleness: The Possibilities of General Purpose Learning Algorithms Applied to Parallel Rule-Based Systems. Machine Learning: An Artificial Intelligence Approach, Vol.2, pp593-623

[5] Niven J (1992) The application of learning systems to electro mechanical engineering - workshop report. C\&I Committee Sec., SERC

|6́ Fogarty T C (1989) edited by Rzevski G Learning new rules and adapting old ones with the genetic algorithm. Artificial Intelligence in Manufacturing, pp275-290 
[7] Fogarty T C (1990) edited by Gottlob G \& Nejdl W Adaptive rule-based optimisation of combustion in multiple burner installations. Expert Systems in Engineering: Principles and Applications, pp241-8

[8] Fogarty T C, ireson N S \& Battle S A (1992) Developing rule-based systems for credit card applications from data with the genetic algorithm. IMA Journal of Mathematics Applied Business and Industry, Vol.4, No.1, pp53-9

[9] CIRIA (1987) Feasibility Study on the Application of Advanced Robotics to Civil Engineering and Construction. Report to the D'TI

[10] Radivisky R A \& Garas F K (1988) Advanced Robotics in the Field of Survey/Inspection Maintenance and Repair of Buildings and Structures. 5th International Symposium on Automation and Robotics in Construction, Tokyo, Japan

[11] HMSO (1988) Black Spot Construction: A Study of Five Years Fatal Accidents in the Building and Civil Engineering Industries. Health and Safety Executive

[12] Cusack M M \& Thomas J G (1991) Exploration and Innovation in Robotics. Advance, Issue 2, Vol.1, Autumn

[13] Cusack M M \& Thomas J G (1992) Wall Climbing Robots for the Inspection and Maintenance of Buildings. 9th International Symposium on Automation and Robotics in Construction, Tokyo, Japan 